Review

\title{
A critical review on the bio-removal of hazardous heavy metals from contaminated soils: Issues, progress, eco-environmental concerns and opportunities
}

\author{
Gang Wu ${ }^{a}$, Hubiao Kang ${ }^{a, e}$, Xiaoyang Zhang ${ }^{c}$, Hongbo Shao ${ }^{b, c, d, *}$, Liye Chu ${ }^{c}$, Chengjiang Ruan ${ }^{\mathrm{d}}$ \\ a State Key Laboratory of Urban and Regional Ecology, Research Center for Eco-environmental Sciences, Chinese Academy of Sciences, Bejing 100085, China \\ b State Key Laboratory of Soil Erosion and Dryland Farming on the Loess Plateau, \\ Institute of Soil and Water Conservation, Chinese Academy of Sciences, Yangling 712100, China \\ ' Institute for Life Sciences, Qingdao University of Science E'Technology (QUST), Qingdao 266042, China \\ d Yantai Institute of Costal Zone Research, Chinese Academy of Sciences, Yantai 264003, China \\ e Graduate University of Chinese Academy of Sciences, Beijing 10049, China
}

\section{A R T I C L E I N F O}

\section{Article history:}

Received 26 July 2009

Received in revised form

21 September 2009

Accepted 22 September 2009

Available online 28 September 2009

\section{Keywords:}

Heavy metals

Soil

Phytoremediation

Microremediation

Eco-environmental concerns

Perspective

\begin{abstract}
A B S T R A C T
Mechanism of four methods for removing hazardous heavy metal are detailed and comparedchemical/physical remediation, animal remediation, phytoremediation and microremediation with emphasis on bio-removal aspects. The latter two, namely the use of plants and microbes, are preferred because of their cost-effectiveness, environmental friendliness and fewer side effects. Also the obvious disadvantages of other alternatives are listed. In the future the application of genetic engineering or cell engineering to create an expected and ideal species would become popular and necessary. However, a concomitant and latent danger of genetic pollution is realized by a few persons. To cope with this potential harm, several suggestions are put forward including choosing self-pollinated plants, creating infertile polyploid species and carefully selecting easy-controlled microbe species. Bravely, the authors point out that current investigation of noncrop hyperaccumulators is of little significance in application. Pragmatic development in the future should be crop hyperaccumulators (newly termed as "cropaccumulators") by transgenic or symbiotic approach. Considering no effective plan has been put forward by others about concrete steps of applying a hyperaccumulator to practice, the authors bring forward a set of universal procedures, which is novel, tentative and adaptive to evaluate hyperaccumulators' feasibility before large-scale commercialization.
\end{abstract}

\section{Contents}

1. Introduction

2. Mechanisms of four metal-removing-methods

2.2. Mechanism of animal remediation

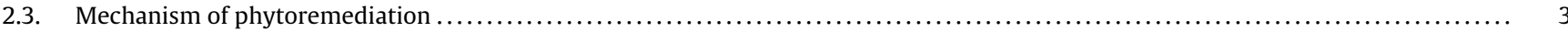

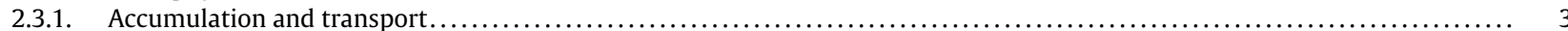

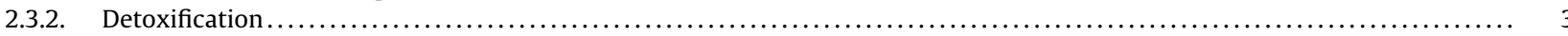

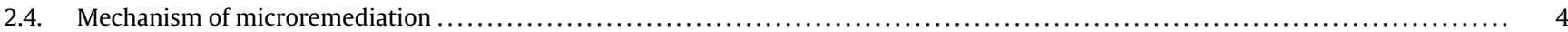

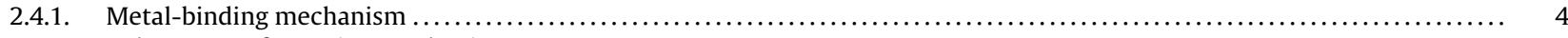

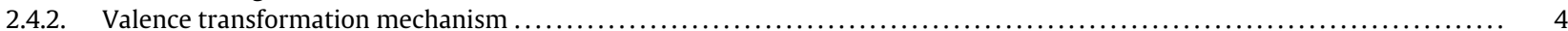

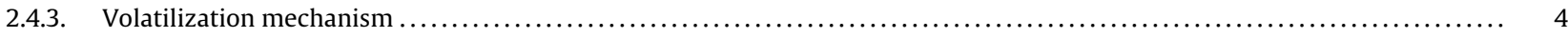

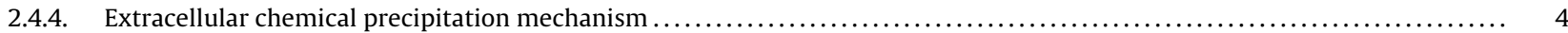

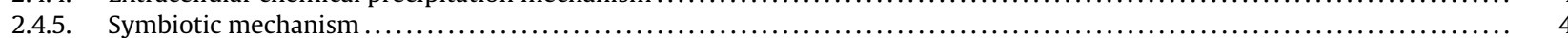

\footnotetext{
* Corresponding author at: Institute for Life Sciences, Qingdao University of Science \& Technology (QUST), Qingdao 266042, China. Tel.: +86 53284023984

E-mail address: shaohongbochu@126.com (H. Shao).
} 






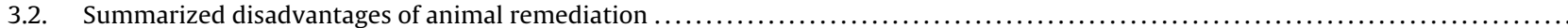

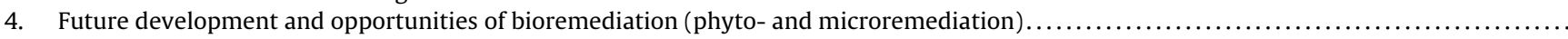

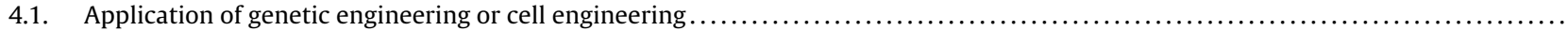



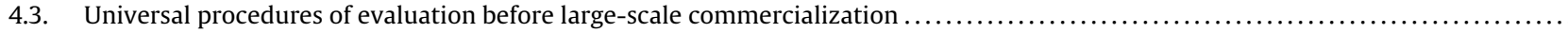

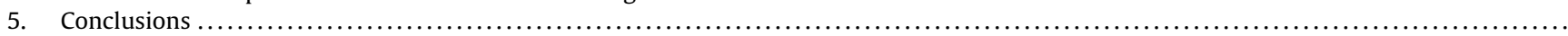

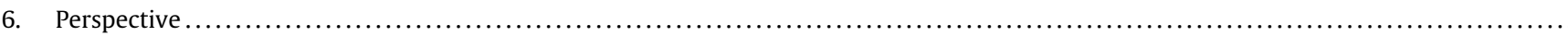

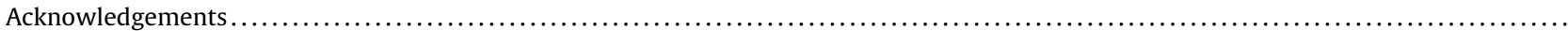

\section{Introduction}

Since the industrial revolution, anthropogenic impacts have caused more and more hazardous heavy metals releasing to environment. Soils, being the basic and most essential part of the ecological system, are heavily contaminated, too. Until now over $20,000,000$ acres of farmland in China have been contaminated by heavy metals such as $\mathrm{Sn}, \mathrm{Cr}, \mathrm{Pb}$, and $\mathrm{Zn}$, which account for almost one fifth of the total arable farmland area. Every year China suffers a 10,000,000 tons' loss of crop output due to deteriorating heavy metal pollution [51]. Different from other organic pollutants, hazardous heavy metals are indestructible, as they cannot be chemically or biologically degraded. Even worse, some heavy metals can concentrate along the food chain and eventually accumulate in human body because we are at the top of the food chain [1-3]. Therefore increasing attention has been paid in recent years to the remediation of polluted soils, among which the use of plants and microbes to remove hazardous metal ions is particularly emphasized [4-6].

Phytoremediation is the use of plants to remove pollutants from environment, while microremediation refers to the use of microbes. Usually if a plant can accumulate more than $1000 \mathrm{mg} / \mathrm{kg}$ (or $1000 \mathrm{ppm}$ ) of $\mathrm{Cu}, \mathrm{Co}, \mathrm{Cr}$, Ni or $\mathrm{Pb}$, or more than $10,000 \mathrm{mg} / \mathrm{kg}$ (or $10,000 \mathrm{ppm}$ ) of $\mathrm{Mn}$ or $\mathrm{Zn}$, it is defined as a hyperaccumulator $[7,8]$. Microbes have a larger specific surface area and are more efficient to activate and remove heavy metals. Plants, however, have harvestable stem and leaves aboveground, which is convenient for subsequent post-processing. Using a transgenic technology to combine the two methods, namely symbiotic system, would be an optimum way to remove and collect hazardous metals efficiently [9-11].

However, transgenic methods sometimes cause gene flow (the transfer of alleles of genes from one individual to another), which is latent but dangerous for a nonnative gene segment by spreading $[12,13]$. Several suggestions of minimizing the resulting potential gene pollution are available in this article. The authors think no country would risk their crop output to plant noncrop hyperaccumulators. So the "cropaccumulators" stand for the developmental tendency of hyperaccumulator plants. In the end, a novel and tentative plan is given by the authors to evaluate the feasibility and demonstrate what procedures are needed before large-scale commercialization.

\section{Mechanisms of four metal-removing-methods}

To date, main four methods were proposed by researchers: chemical or physical remediation, animal remediation such as earthworm, phytoremediation and microremediation. Because of the obvious disadvantages and deficiency in feasibility, wide application of the former two methods is restricted. Summarized aspects of disadvantages of these two methods are given in Section 3. In this part, mechanism of each four methods for removing hazardous heavy metal is explained and compared, and we concerns more about the latter two-phytoremediation and microremediation (bioremediation).

\subsection{Mechanism of chemical or physical remediation}

Chemical or physical method is early used and even endemically commercialized in America. Physical methods (e.g. soil leaching method and absorbent fixation) and chemical methods (e.g. bioreduction and chelate extraction) are used in practice. In these methods the use of chelators cannot be avoided. By adding synthetic chelators such as EDTA (ethylenediamine-tetracetic acid), both the solubility and bioavailability of heavy metals are improved. A chelating reagent's molecule can form several coordinative bonds to a certain metal atom, increasing its concentration in soil aqueous phase and mobility (Fig. 1) [1]. Considering some metal ions strongly bonds to the soil phase and are less bioavailable, powerful chelating reagents are employed such as Na salt of EDTA. However, such approach needs not only expensive chemical reagent and machines but also many technicians. Worse, excessively usage of chemical chelates has been proven to pollute the ground water and negatively affect soil quality, for many necessary ions are also chelated unselectively. For example, elements Fe and Ca are usually lost after the spray of EDTA because their concentration in the soil is much higher than those target heavy metals such as $\mathrm{Pb}$ and thus have more access and possibility to chelation. Wenzel et al. [1] conducted an experiment using canola (Brassica napus $\mathrm{L}$ ) and reported that leaching losses of $\mathrm{Cu}, \mathrm{Pb}$ and $\mathrm{Zn}$ (polluting ground water) far exceeded the amounts of metal taken up by plants after EDTA was applied, which indicated that under some certain circumstance the disadvantage of chelating reagent far outweigh its advantage. Therefore, taking reagent toxicity, unselectivity and inefficacy into account, a careful consideration concerning ecology, economy and human health is imperative before chelators are being put into practice [14].

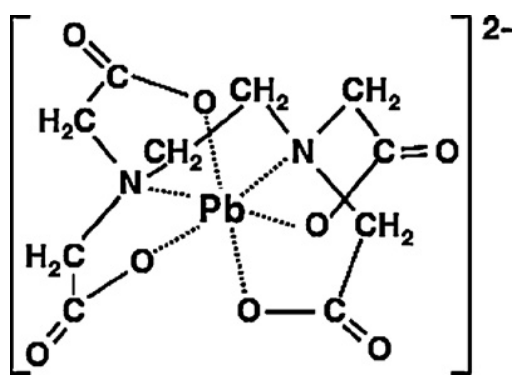

Fig. 1. EDTA-Pb complexes. Dotted bonds to $\mathrm{Pb}$ are coordinate. 


\subsection{Mechanism of animal remediation}

Animal here mainly refers to earthworm because it is one of the most important soil organisms and plays an indispensable role in improving soil quality [15-18]. By their feeding, burrowing, excreting and metabolic redox material, both soil texture and nutrition content are improved. Chemical groups such as $-\mathrm{COOH}$ and $-\mathrm{CO}$ are generated and exuded, which acidify soil and activate heavy metals. Several kinds of gel material are also excreted which facilitate complexion and chelation of metal ions. However, because of the relatively small amount and specific surface area compared with microbes, such improvement is neither notable nor stable. According to Baker et al. [19], after Eisenia foetida earthworm was inoculated, $\mathrm{pH}$ of a cock manure decreased by $0.7-0.9$. However, if the inoculation occurred in an acidic red soil the $\mathrm{pH}$ value drops only by $0.03-0.18$; if the inoculation happens in a sandy soil, no obvious decrease of $\mathrm{pH}$ is observed. Thus current studies imply that the effectiveness and efficiency of earthworm depend too much on outer conditions and may not be the optimum way of rapidly removing heavy metals. Further investigation in this field is needed.

\subsection{Mechanism of phytoremediation}

\subsubsection{Accumulation and transport}

In the rhizosphere of hyperaccumulator plants, protons are released by root to acidify the soil, which mobilize metal ions and increase metal bioavailability. This mechanism is supported by Crowley et al. in 1991 [2]. However, due to metal ions' charge, lipohilic cellular membrane would be the first barrier of ions' entrance into cells. Fortunately, the following kinds of secretion can facilitate the transportation process.

(1) Transporter proteins: Specific binding domain is existed in such proteins, which binds to and transports metal ions from extracellular space into cells. Lasat et al. [3] have found that hyperaccumulator Thlaspi caerulescens had bigger capacity for $\mathrm{Zn}^{2+}$ than its relative $T$. arvense. And such gap is caused by different amounts of $\mathrm{Zn}$ transporter proteins [5], which indicates that transporter proteins play a crucial role.

(2) Nature chelators: As we know chelators such as EDTA can bind to heavy metal ions and make them render uncharged. An uncharged ion is of high mobility and is much easier to get through cellular membrane. In fact, plants can excrete nature chelators, which is much less toxic and more biodegradable as compared to EDTA (Table 1 ).

Among nature chelators, phytochelatin (PC) and metallothionein (MT) interest many scientists and is well studied. MTs are categorized into three classes: Class 1 MTs referred to polypeptides related to mammals, which contain 61 amino acids but lack aromatic amino acid or histidines; Class 2 MTs originally come from yeasts, and Candida albicans or cyanobacteria [6]; an familiar chelator belonging to this class is Saccharomyces cerevisiae MT, contributing to plants' high copper tolerance [7]; Class 3 MTs is PCs exactly, which are composed of only three amino acid-Glu, Cys and Gly, with Glu and Cys residues linked through a $\gamma$-carboxymide bond. In addition, Kagi [7] have found that heavy metals such as $\mathrm{Cd}, \mathrm{Zn}, \mathrm{Hg}, \mathrm{Ag}$ and $\mathrm{Pb}$ can induce the

\section{Table 1}

Chelators that are commonly used are of importance.

\begin{tabular}{ll}
\hline Nature chelators & Phytochelatin (PC) metallothionein (MT) \\
& Organic acids \\
Synthetic Chelators & EDTA (ethylenediamine-tetraacetic acid) EGTA \\
& (ethylene glycol tetra acetic acid) DTPA \\
(diethylenetriaminepentaacetic acid)
\end{tabular}

Table 2

Metal ions and their transporters.

\begin{tabular}{ll}
\hline ZNT protein (encoded by ZIP gene) & $\mathrm{Zn}^{2+}$ and $\mathrm{Cd}^{2+}$ \\
Nramp protein (encoded by AtNramp gene) & $\mathrm{Cd}^{2+}$ and $\mathrm{Fe}^{2+}$ \\
Protein encoded by NtCBP4 & $\mathrm{Pb}^{2+}$ \\
Aquaglyceroprins & $\mathrm{As}^{3+}$ \\
Phosphate transporter & $\mathrm{As}^{5+}$ \\
IRT1 (iron-regulated transporter) & $\mathrm{Fe}^{2+}, \mathrm{Mn}^{2+}$, and $\mathrm{Zn}^{2+}$ \\
\hline
\end{tabular}

synthesis of MTs especially in animal and plant species. A recent study shows that the best activator is Cd followed by $\mathrm{Ag}, \mathrm{Bi}, \mathrm{Pb}$, $\mathrm{Zn}, \mathrm{Cu}, \mathrm{Hg}$ and $\mathrm{Au}$ [9].

(3) Organic acids: Several organic acids (e.g. malic acid and citrate) have been identified as positive bio-reagents to accelerate the absorption of heavy metals by root. Such mechanism is even more notable in the root-shoot transportation.

However, substantial achievements are lacked in the root-shoot transportation except two points: one is that root-shoot pathway is closely related to plants' transpiration efficiency; the other is one of chelator ligands (histidine) is found in high levels in the xylem sap of Ni tolerant plant (Alyssum lesbiacum) and the coordination of Ni with histidine is substantiated by Kramer et al. [10], which implies that chelation mechanism also works in the process of xylem transferring.

On the molecular level, accumulation and transport mechanism is partly clarified. Many transporters encoded by specific genes are investigated and it is common that one kind of metal ion can be transported by different carriers (Table 2) [20-27].

\subsubsection{Detoxification}

As we know some hazardous heavy metals exercise a detrimental influence on cells by binding to vital protein, interfering with cellular activities and inhibiting regulation of cells. Luckily, hyperaccumulator plants have evolved their own mechanisms to protect themselves from negative heavy metal stress. Several important detoxification mechanisms are explained as follows:

(1) Chelation: Chelation plays a crucial role not only in the accumulation and transportation of heavy metals but also in the detoxification phase. Usually chelators have ligands (most commonly histidine and citrate) and can bind metal ions. Combined metal ions appear uncharged and inert to react to other substance, by which way heavy metals' damage towards cell is reduced significantly.

(2) Vacuolar compartmentalization: Since vacuole is widely considered as the main storage place of heavy metals in plant cells, vacuolar compartmentalization is quite effective in controlling the distribution and concentration of metal ions. To compartmentalize vacuole is to "arrest and imprison" hazardous metal ions, constricting them into a limited site. Thus other parts of the cell have no access to those dangerous metal ions and safety is of course ensured. This mechanism is proved to be true in the Cd detoxification and tolerance by Salt et al. [11]: Cd induces the synthesis of PCs and then forms a Cd-PC molecule, which will be transferred into the vacuole by a $\mathrm{Cd} / \mathrm{H}$ antiport and an ATPdependent PC-transporter. Additionally Kramer et al. [12] have reported that by "imprisoning" most of the intracellular Ni into vacuole, metal tolerance of hyperaccumulator $T$. goesingense is greatly improved, which confirms the compartmentalization theory, too.

(3) Volatilization: By converting metal ions into volatile state, some plant species avoid the lasting damage caused by accumulation and long-time stay of heavy metals. A representative example is the bioprocess of $\mathrm{Hg}$, which is a worldwide volatile pollutant and which is able to accumulate in human bod- 
ies. However, not all the plants possess such ability and even among those innate $\mathrm{Hg}$-resistant species, the relatively small amount of accumulation and their spatial distribution have greatly limited their wide cultivation. Thus scientists have employed genetic engineering and several transgenic plants have showed satisfactory performance to convert and volatilize metals. Transgenic species expressing organomercurial lyase (MerB) have much higher tolerance to organic $\mathrm{Hg}$ complex than wild type and can convert methylmercury to $\mathrm{Hg}(2)$, which is 100 times less toxic than the former one [13]. Furthermore, transgenic plants expressing both MerA (enzyme that reduces $\mathrm{Hg}(2)$ to $\mathrm{Hg}(0))$ and MerB have shown the highest tolerance to organic $\mathrm{Hg}$ (up to $10 \mu \mathrm{M}$ ) compared with MerB species' $5 \mu \mathrm{M}$ and wild type's $0.25 \mu \mathrm{M}$ [14].

\subsection{Mechanism of microremediation}

\subsubsection{Metal-binding mechanism}

Three substances should be mentioned for this mechanism: MT, PC, and some novel metal-binding peptides. As we know from the former part of this article, MT and PC play a crucial part in plant-metal interaction. In fact, in the microbial world such interplay also exists. By binding to heavy metal ions MTs facilitate microbes' absorption or transportation of metal ions, and so do PCs, which are composed by only three amino acids (Gly, Cys and Gly). That over expression of PC synthase in microbes is effective to the accumulation and tolerance of metal ions has been reported by Sriprang et al. [15]. By expressing the Arabidopsis thalina gene encoding PC synthase, enhanced Cd accumulation is observed in Mesorhizobium huakuii subsp. rengei B3 and Escherichia coli cells. Recent years novel metal-binding peptides containing histidines or cysteines have been found and engineered. These peptides are usually of higher affinity, specificity and selectivity for a certain metal ion. Related and in-depth study, however, is scarce.

\subsubsection{Valence transformation mechanism}

Metals of different valencies vary in toxicity. By excreting special redox enzyme, plants skillfully convert hazard metals to a relatively less toxic state and decrease possible metal stress and damage. For example, reduction of $\operatorname{Cr}(6)$ to $\operatorname{Cr}(3)$ is widely studied, the latter one of which is both less mobile and less toxic. Additionally, Kashiwa [16] has found that Bacillus sp. SF-1 was good at reducing high concentration of $\mathrm{Se}(6)$ into elemental Se. The most persuasive example of this mechanism is the mercury-resistant bacteria, in which organomercurial lyase (MerB) is produced. As we see from Fig. 2, methylmercury is converted to $\mathrm{Hg}(2)$, which is 100 -fold less toxic than the former one [27-29].

\subsubsection{Volatilization mechanism}

By turning metal ions into volatile state, microbes escape possible negative effect that dangerous metal ions bring them. However, such approach is feasible for only a few metals such as $\mathrm{Hg}$ and metalloid Se. For the majority of most other metals which have no volatile state at natural conditions, this pathway is closed. To date, the way microbes deal with element $\mathrm{Hg}$ is relatively clear. In the cells of mercury-resistant bacteria there is a MerA enzyme, an enzyme that reduces $\operatorname{Hg}(2)$ to volatile form $\operatorname{Hg}(0)$ [16,17] (Fig. 3).

$$
\mathrm{R}-\mathrm{CH}_{2}-\mathrm{Hg}^{+}+\mathrm{H}^{+} \quad \stackrel{\mathrm{MerB}}{\longrightarrow} \mathrm{R}-\mathrm{CH}_{3}+\mathrm{Hg}(\mathrm{II})
$$

Fig. 2. MerB converting organic $\mathrm{Hg}$ to $\mathrm{Hg}(2)$.

$$
\mathrm{RSHg}^{+}+\mathrm{NADPH} \stackrel{\mathrm{MerA}}{\longrightarrow} \mathrm{Hg}(0)+\mathrm{RSH}+\mathrm{NADP}^{+}
$$

Fig. 3. MerA converting $\mathrm{Hg}(2)$ to $\mathrm{Hg}(0)$ and facilitating volatilization.

\subsubsection{Extracellular chemical precipitation mechanism}

Quite a number of binding substances were excreted by microbes, ranging from simple organic acid, alcohols to large polysaccharides, humic and fulvic acids. In fact, not only metals but also metal sulphides and oxides can be entrapped and absorbed by an extracellular mixture of polysaccharides, mucopolysaccarides and proteins [24]. Recent studies have found that peptidoglycan carboxyl groups are the main cation binding sites for Gram-positive bacterial cell walls while phosphate group for Gram-negative microbes and chitins for fungi. No matter whether the precipitation happens in the outer surface of the cell wall or away from it, this mechanism is successful by keeping harmful metal ions out of cytoplasm [25].

\subsubsection{Symbiotic mechanism}

A big disadvantage of microremediation is that absorbed heavy metals would still stay in the soil, so symbiotic mechanism would be more effective by combining both microremediation and phytoremediation. Due to the symbiotic microbes' large amount and specific surface area, binding reagent such as MTs, PCs and organic acid will be excreted more by symbiotic systems than by sole plants. Thus soil will be improved with better acidification, which ultimately leads to a better solubility, mobility and bioavailability of heavy metals. After heavy metal particles are activated, the subsequent process can be divided into two ways. One is that metal ions are accumulated by plants root, transported in the xylem and detoxified through chelation, vacuolar compartmentalization and volatilization, just as normal phytoremediation does. The other way is heavy metals will be accumulated in rhizosphere and nodules. Rhizosphere bacteria's essential role in achieving optimum rates of selenium accumulation and volatilization has been proven by Yang et al. [21] in an Indian mustard experiment. Another symbiotic experiment was provided by Sriprang et al. [18] by inserting the MTL4 gene into M. huakuii subsp. rengei B3. Data showed that the symbionts $\mathrm{Cd}^{2+}$ absorption increased by 2.3-6.6-fold in nodules while obvious accumulation of $\mathrm{Cu}^{2+}$ was not observed.

\section{Evaluation of four metal-removing-methods}

\subsection{Summarized disadvantages of chemical/physical remediation}

(1) It is an expensive and labor-intensive way. Using large machine and synthesizing a great amount of chelators could be costly and the application of landfilling and leaching technology demands not only professional technicians but also many hours. Someone has estimated that in order to reduce soil $\mathrm{Pb}$ concentration from $1.4 \mathrm{~g} / \mathrm{kg}$ to $0.4 \mathrm{~g} / \mathrm{kg}$ in ten years, phytoremediation would cost only $\$ 27,900$. Compared with landfilling method's $\$ 1,620,000$ and soil leaching method's $\$ 790,000$, phytoremediation is very economical.

(2) Natural soil' structure, texture and fertility can be impaired by the method itself and by the reagent added.

(3) Excessive use of chelators would poison both plants and microbes. The most widely used chelator EDTA is both toxic and nonbiodegradable.

(4) Chemical/physical method may lead to pollution by mobile heavy metal ions leaching into ground water, since the use of binding reagents makes metal ions more soluble and mobile.

(5) Chelators such as EDTA are usually lack of selectivity and easily cause beneficial ions' loss (especially for Fe and Ca). 
Table 3

Genes introduced into plants and their source and target. G+ stands for Gram-positive bacteria and G- stands for G-negative ones.

\begin{tabular}{|c|c|c|c|c|c|c|c|}
\hline Gene & Product & Source & Target & Gene & Product & Source & Target \\
\hline merA & $\mathrm{Hg}(\mathrm{II})$ reductase & $\mathrm{G}^{+}$ & $\begin{array}{l}\text { Liriodendron tulipifera, } \\
\text { Nicotiana tabacum }\end{array}$ & CGS & $\begin{array}{l}\text { Cystathione-gamma- } \\
\text { synthase }\end{array}$ & A. thaliana & B. juncea \\
\hline merA & $\mathrm{Hg}$ (II) reductase & G- & A. thaliana & CSase & Cysteine synthase & Spinach & N. tabacum \\
\hline merB & Organomercurial lyase & G- & A. thaliana & gsh2 & GSH synthase & E. coli & B. juncea \\
\hline merA & $\mathrm{Hg}(\mathrm{II})$ reductase & G- & N. tabacum & gsh1 & $\gamma$-Glu-Cys synthase & E. coli & B. juncea \\
\hline merB & Organomercurial lyase & G- & N. tabacum & gsh1 & $\gamma$-Glu-Cys synthase & E. coli & Populus tremula \\
\hline AtPCSI & PC & A. thaliana & A. thaliana & NtCBP4 & Cation channel & N. tabacum & N. tabacum \\
\hline APSI & ATP sulfurylase & A. thaliana & Brassica juncea & SL & Se-Cys lyase & Mouse & A. thaliana \\
\hline MT-1 & MT & Mouse & N. tabacum & TaPCSI & PC & T. aesitivum & N. glauca \\
\hline
\end{tabular}

\subsection{Summarized disadvantages of animal remediation}

(1) Animals such as earthworm are usually small in total number and specific surface area, which makes them inefficient in absorbing and accumulating heavy metals.

(2) Animals' rigorous demand for comfortable environment and excessive dependence on organic substance limit their wide use and decrease their practicality.

(3) Heavy metals are still in the soil if lack of a feasible method to collect all the earthworms.

(4) Clear mechanism of accumulating and detoxifying heavy metal in animals needs to be investigated by further studies.

\section{Future development and opportunities of bioremediation (phyto- and microremediation)}

\subsection{Application of genetic engineering or cell engineering}

In recent years, genetic engineering technology seems increasingly necessary, because most nature hyperaccumulators are not satisfying (due to its slow growing, low biomass-production and rigorous demand for growing conditions). Aided by this powerful tool, many heavy metal resistant genes have been introduced into plant cells (Table 3) [19]. By over expression of natural chelators (MTs, PCs and organic acid), not only ions' entrance into cell but also translocation in xylem and other part is facilitated; by excreting certain transporter proteins, specific ions was bound and transferred; by encoding special oxidoreductase such as MerA and MerB, heavy metals' valence is changed into a less toxic one or a more volatile one; by horizontal transfer of plasmid, resistant gene are exchanged among rhizosphere bacteria and this benefits bacteria's accumulating and transferring metal ions.

Cell engineering technology (such as cell fusion or somatic hybridization) also shows great power. Because polyploid plants are usually bigger in size and more active in transpiration, which does good to the transportation of heavy metals in root-to-shoot process, this method is of great significance (Fig. 4) [23]. Hybrid (B) not only has a bigger size than its parent Brassica juncea (A) and Thlaspi caerulescens $(C)$, but also better hyperextraction in absorbing $\mathrm{Pb}, \mathrm{Ni}$ and $\mathrm{Zn}$. The amount of $\mathrm{Pb}$ absorbed by hybrid $(\mathrm{B})$ is almost the sum of its parents (A) and (C) [20].

However, a big disadvantage coming with transgenic technique is the genetic pollution caused by, for example, the floating of transgenic pollen and the horizontal transferring of plasmids among microbes. In addition, because both pollen and plasmid is invisible to naked eyes, not until the horrible mutant species grow up and cause severe aftermath can we realize their damage. Therefore biosafety aspects must be taken into account and application of transgenic species need domestic legislation.

Here the authors contribute three feasible suggestions that minimize the potential damage caused by genetic pollution:
(1) Choosing self-pollinated plants.

In a self-pollinated plant, pollen moves to the female part of the same flower or to another flower on the same individual plant. And this means fertilization is completed within a closed environment. Therefore, self-pollination would be an effective way to avoid the spreading of transgenic genes into other species.

(2) Using cell engineering technology to create infertile polyploid species.

Infertile plants cannot transfer any gene to the next generation and so potential spread of gene segment becomes impossible. Technically two species are feasible: one is allopolyploid (the combination of two heterogeneous sets of chromosomes) and the other is triploid. Both approaches are able to make plants infertile by lack of equation-division mechanism of chromosomes.

(3) Among microbes, transgenic technique should be limited within the sphere of rhizosphere bacteria and symbiotic bacteria, because such kind of microbe mainly congregates around the root and shows a very slight distribution outside the rhizosphere. In detail, the population of "endophytic bacteria" living within the plant tissues is easier controlled than that of rhizospheric bacteria living on or round the plant roots, because the former depend more on plants. Therefore by restricting the transgenic and foreign genes in a limited area and a finite amount, potentially harm of genetic pollution is minimized.

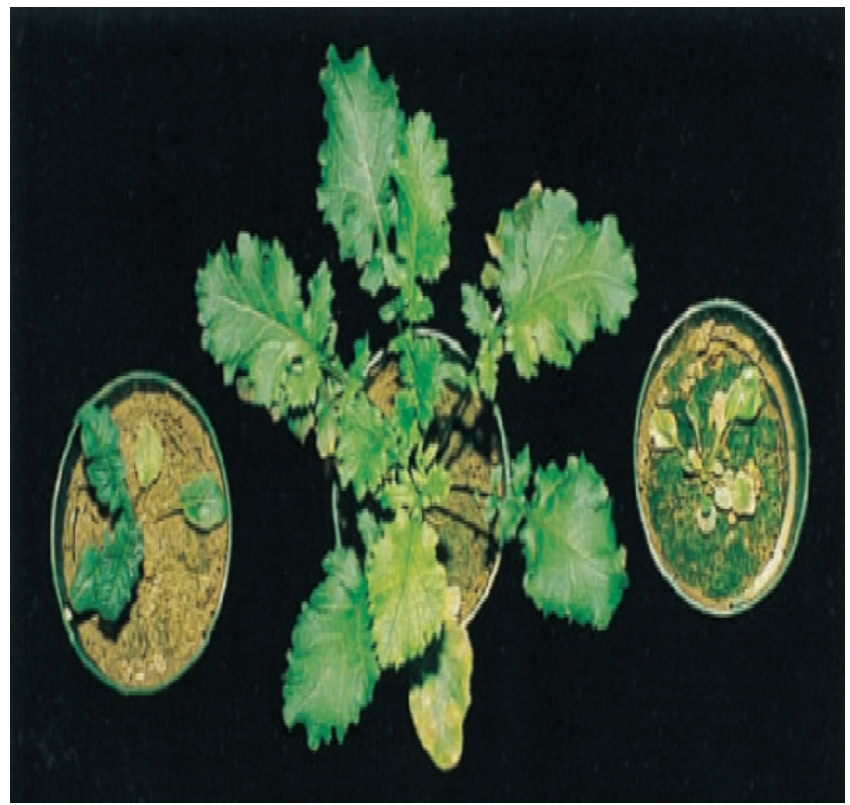

Fig. 4. Somatic hybrid (B) and its parents (A) and (C). 


\subsection{The development of crop hyperaccumulators}

Another new viewpoint towards phytoremediation is that adequate attention must be paid to the development of crop hyperaccumulators, termed by the author as "cropaccumulators", such as wheat, maize and rice [30-33]. In other words, investigations of other "noncropaccumulators" species would be of little significance in application. A large area of farmland in the world is contaminated by heavy metals and need remediation. Considering the finite and precious farmland sources and big population, no country would stop growing crops and turn to noncrop hyperaccumulators which do not provide grain. Furthermore, it usually takes several years to completely remove a certain heavy metal from soil. This makes the application of noncrop hyperaccumulators more unrealistic. After all, no country could afford zero harvest and even famine for several years.

To date, more than 400 plant species have been identified as metal hyperaccumulators and the best hyperaccumulator varies according to regions with different climate and soil types. To date, the most studied and promising species is within Cruciferae family such as Brassica genus, Alyssuns genus and Thlaspi genus, but $75 \%$ of them are Ni tolerant which grow on ultramafic soils $[23,24]$. Unfortunately almost none of them can be used as crops in the farmland. Thus it is critical that we paid immediate attention to the "cropaccumulators" field. We recommend two ways to develop this "cropaccumulators" species:

(1) By transgenic approach: Through the introduction of foreign resistant genes into crops, capability for accumulating, transporting and detoxifying heavy metals can be significantly improved. Even if the improvement is not as notable as expected, it does not matter because "cropaccumulators" can be grown every year as normal field crops and heavy metals are bound to be removed thoroughly after a long period of time. As long as heavy metals do not accumulate in the edible part such as grain, long-time growing of "cropaccumulators" would not reduce the total crop outcome and impair the grain quality.

(2) By symbiotic approach: Finding an appropriate kind of symbiotic bacteria is the key of this method. In a symbiotic system, heavy metals are more likely to be accumulated in nodules. This is good for us because generally crops bear grains aboveground and the fear that heavy metals may enrich in edible part is eliminated. Another big advantage is that some microbes produce antibiotics to enhance plants immunity and some produce necessary nutrients and even plant growth hormones [25-32]. Therefore the future of this method would be bright.

\subsection{Universal procedures of evaluation before large-scale} commercialization

As we discussed in Section 4, "cropaccumulators" created by genetic or cell engineering technology will of great importance and hope. However, related literature concerning concrete steps of applying a hyperaccumulator to practice is seldom found. The author tentatively puts forward a new evaluation plan which tells us clearly what should be done before large-scale commercialization of a new hyperaccumulator species (Fig. 5).

Explanations of each step in Fig. 5.

Step 1: After a transgenic or symbiotic species is created, theoretical biosafety assessment is indispensable. Since foreign genes would enter the ecological system automatically and cannot be easily removed once a seed is planted. Based on experience and hermetic experiments biosafety can be estimated. The following parameters are recommended: sexual compatibility of pollens, blossom and spatial dis-

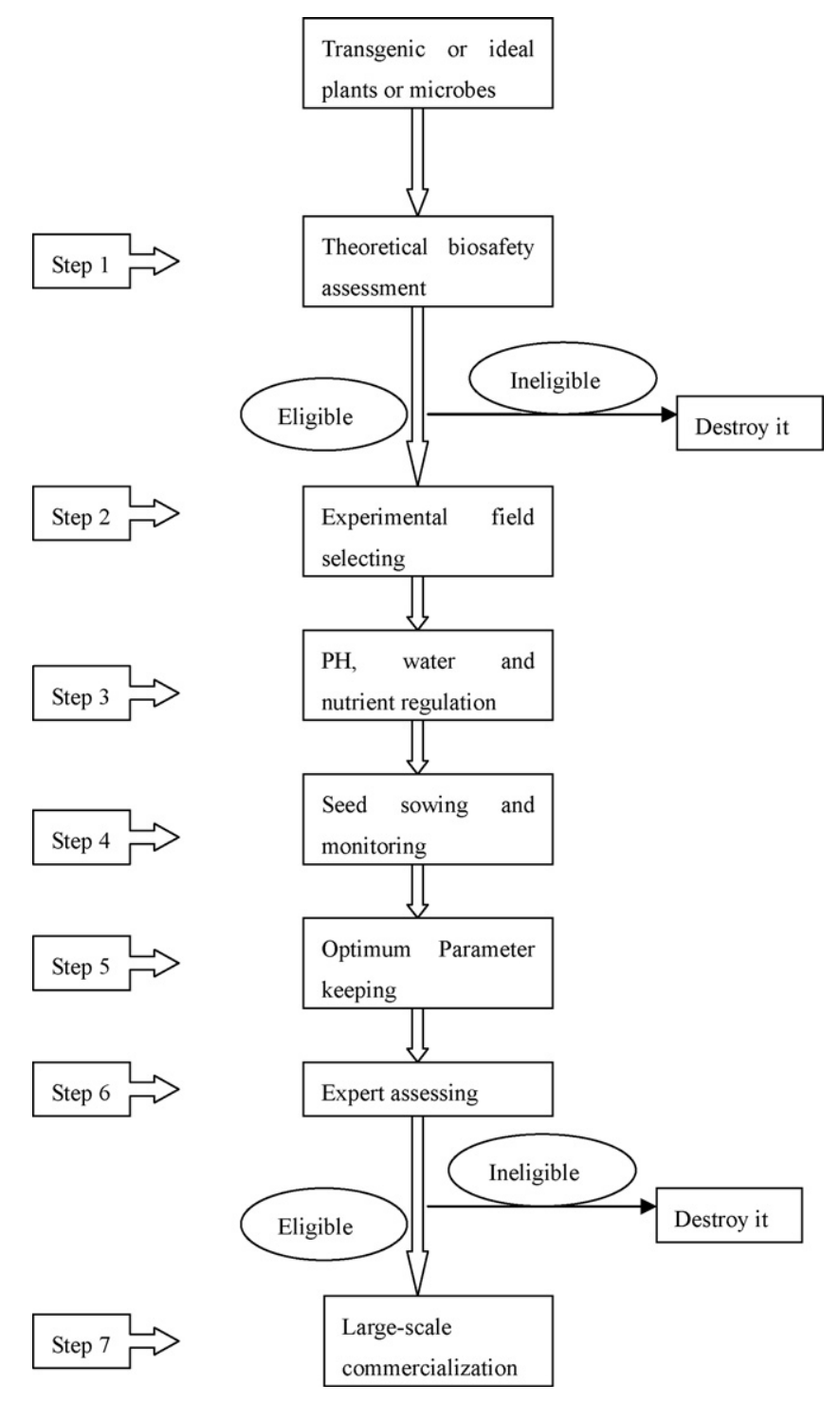

Fig. 5. Universal procedures of evaluation before large-scale commercialization.

tribution of nearby plants, and the fertility of the next generation. If the new species is eligible and biosafe to the ecology, it is allowed to go to the next step. If not, simply destroy it (for the purpose of eliminating nonnative genes).

Step 2: Experimental field selected should be representative. A relatively closed environment is necessary to minimize gene flow phenomenon. If possible the selected field should be also easily regulated in water, $\mathrm{PH}$, nutrient, and sampling for the subsequent steps.

Step 3: As we know soil acidity is closely related to the activation and accumulation of heavy metal. Nutrient, however, also matters. Studies show that a proper amount of $\mathrm{N}$ and $\mathrm{K}$ promote the uptake of $\mathrm{Pb}$ but a large amount inhibits the $\mathrm{Pb}$ absorption. The addition of $\mathrm{P}$ does not improve the accumulation of $\mathrm{Pb}$, which even counteracts the original absorbing [28]. Water supplying relates with transpiration, which plays a great role in the transportation of the heavy metals. Every plant and microbe has its optimum temperature, $\mathrm{pH}$, water content and nutrient supplying for living, and satisfying these demands is important.

Step 4: We recommend natural outdoor field with sunlight to sow the new test species and spring would be the best season for the most species. Although simulated lab field can 
provide similar conditions, it is not suitable for this evaluation process in many aspects especially in the assessment of genetic pollution where interaction with other plants is unavoidable. Besides, another important aim of step 4 is to monitor how well the plant or symbiotic system survives while removing heavy metals. Several parameters are cited here, which can be categorized into two classes. One is chemical method which is stable, and the other one is biological parameters which are less stable but more effective and sensitive. For example, chemical parameters can show the total amount of a certain metal, which may be useless because only soluble or biodegradable forms of metal ion can be absorbed. A government law (National Standard of the People's Republic of China) provides ways of heavy metal measurement, which is accurate and legal. Micro-parameters reflect not only heavy metal content and stress, but also compatibility between bacteria and plants. Representative parameters include the ratio of microbial biomass $C\left(C_{\text {mic }}\right) / C\left(C_{\text {org }}\right)$ and metabolic quotient $\left(q \mathrm{CO}_{2}\right)$ in biochemical level and CLPP in community level. Usually when heavy metal concentration goes up, decrease in $C / C$ ratio and increase in metabolic quotient can be observed.

Step 5: Keeping parameters at an optimum value can be achieved by several approaches. To decrease soil $\mathrm{pH}$ value, either $\mathrm{H}_{2} \mathrm{SO}_{4}$ or organic fertilizer can be used. To increase $\mathrm{PH}$, lime or other alkaline substance is useful. By regulating water supplying, both transpiration and Eh value (a redox index of soil) is controlled. The use of element $\mathrm{P}, \mathrm{N}$ and $\mathrm{K}$ can also affect those parameters. Through step 5's regulation we try to ensure that new hyperaccumulators work at a normal and relatively high efficiency.

Step 6: Different from Step 1, expert assessment is practical because plants have matured. Maturity means pollens have been produced and spread. Therefore at this time biosafety issue is quite realistic. A detailed and rigorous environmental risk assessment must be completed to avoid genetic pollution as much as possible. Other index should also be assessed such as biomass, total cost of the growing, and growth rate. Besides these, experts need to estimate how long bioremediation would take, for an excessively long duration is not suitable for process feasibility. If the plant is qualified in this step, it can go to further large-scale commercialization; if not, destroy it (for the purpose of eliminating nonnative genes).

Step 7: The performance of the last step needs a national legislation concerning transgenic species and its potential risk. If it is a novel symbiotic system using no transgenic technology, such procedure is not needed. Additionally, when commercializing, different kinds of hyperaccumulators can be grown by turn since one kind of hyperaccumulators is generally good at dealing with finite kinds of metal.

\section{Conclusions}

(1) Bioremediation has more advantages and should be focused more, especially on transgenic methods.

(2) Future transgenic technology should focus on four promising targets: over expression of natural chelators (MTs, PCs and organic acid), transporter proteins, special oxidoreductase and resistant gene in symbiotic microbe.

(3) Several ways of minimizing genetic pollutions are put forward such as choosing self-pollinated plants, creating infertile polyploid species and carefully selecting easy-controlled microbe species.

(4) Crop hyperaccumulators should be paid more attention for studies on noncrop species are of limited use.
(5) Universal procedures are put forward to evaluate hyperaccumulators' feasibility before large-scale commercialization.

\section{Perspective}

Compared to chemicophysical method, bioremediation shows great advantage. Studies on this field are increasing and its mechanism is more and more clear [33-41]. Using transgenic technology is a tendency in the future to create an ideal species purposely. However, genetic pollution must be taken into consideration, which can be avoided or minimized by using methods mentioned above.

In the future crop hyperaccumulators will be a better choice due to its feasibility, in the field of which current emphasis is scarce. Microbes, in many cases, are more efficient in accumulating and absorbing heavy metals because of their astronomical amount and specific surface area. Furthermore, technique of genetic engineering in microbes is easier and more mature than in plant cells. Therefore, using transgenic technology to create an optimum plant + soil + microbes combination would be a promising way in the future development [42-50].

As to the estimation of a new species or symbiotic system, Fig. 5 offers a feasible risk assessment plan and may accelerate the application process.

\section{Acknowledgements}

This work was jointly supported by One hundred-Talent Plan of Chinese Academy of Sciences, 973 Program of China (2007CB106803), the CAS/SAFEA International Partnership Program for Creative Research Teams, the CAS-local government Cooperative Project, the Cooperative \& Instructive and Awarding Foundation of State Key Laboratory of Soil Erosion and Dryland Farming on the Loess Plateau (10501-HZ; 10501-JL). We expressed sincere thanks for 3 reviewers and Editor, Dr. Too Hwa Tay for their suggestive comments and making the paper improved greatly!

\section{References}

[1] W.W. Wenzel, et al., Chelate-assisted phytoextraction using canola (Brassica napus L) in outdoors pot and lysismeter experiments, Plant Soil 249 (2003) 83-96.

[2] D.E. Crowley, et al., Mechanisms of iron acquisition from siderophores by microorganisms and plants, Plant Soil 130 (1991) 179-198.

[3] M.M. Lasat, et al., Physiological characterization of root $\mathrm{Zn}^{2+}$ absorption and translocation to shoots in Zn hyperaccumulator and nonaccumulator species of Thlaspi, Plant Physiol. 112 (1996) 1715-1722.

[4] N.S. Pence, et al., The molecular physiology of heavy metal transport in the Zn/Cd hyperaccumulation Thlaspi caerulescens, Proc. Natl. Acad. Sci. U.S.A. 97 (2000) 4956-4960.

[5] R.K. Mehra, et al., Metal ion resistance in fungi: molecular mechanisms and their regulated expression, J. Cell Biochem. 45 (1991) 30-40.

[6] D.R. Winge, et al., Yeast metallothionein: sequence and metal-binding properties, J. Biol. Chem. 260 (1985) 14464-14470.

[7] J.H.R. Kagi, Overview of metallothionein, Methods Enzymol. 205 (1991) 613-626.

[8] S. Loeffler, et al., Termination of the phytochelatin synthase reaction through sequestration of heavy metals by the reaction product, FEBS Lett. 258 (1989) 42-46.

[9] U. Kramer, et al., Free histidine as a metal chelator in plants that accumulate nickel, Nature 379 (1996) 635-638.

[10] D.E. Salt, et al., Cadmium transport across tonoplast of vesicles from oat roots. Evidence for a $\mathrm{Cd}^{2+} / \mathrm{H}^{+}$antiport activity, J. Biol. Chem. 268 (1993) 12297-12302.

[11] D.E. Salt, et al., MgATP-dependent transport of phytochelatins across the tonoplast of oat roots, Plant Physiol. 107 (1995) 1293-1301.

[12] U. Kramer, et al., Prince subcellular localization and speciation of nickel in hyperaccumulator and non-accumulator Thlaspi species, Plant Physiol. 122 (2000) 1343-1353.

[13] S.P. Bizily, et al., Phytoremediation of methylmercury pollution merB expression in Arabidopsis thaliana confers resistance to organomercurials, Proc. Natl. Acad. Sci. U.S.A. 96 (1999) 6808-6813.

[14] S.P. Bizily, et al., Phytodetoxification of hazardous organomercurials by genetically engineered plants, Nat. Biotechnol. 18 (2000) 213-217. 
[15] R. Sriprang, et al., Enhanced accumulation of $\mathrm{Cd}^{2+}$ by a Mesorhizobium sp. transformed with a gene from Arabidopsis thaliana coding for phytochelatin synthase, Appl. Environ. Microbiol. 69 (2003) 1791-1796.

[16] M. Kashiwa, et al., Removal of soluble selenium by a selenate-reducing bacterium Bacillus sp. SF-1, J. Ferment. Bioeng. 83 (2001) 517-522.

[17] B. Fox, et al., Mercuric reductase. Purification and characterization of a transposon-encoded flavoprotein containing an oxidation reduction active disulfide, J. Biol. Chem. 257 (1982) 2498-2503.

[18] R. Sriprang, et al., A novel bioremediation system for heavy metals using the symbiosis between leguminous plant and genetically engineered rhizobia, J. Biotechnol. 99 (2002) 279-293.

[19] A.J.M. Baker, et al., Metal hyperaccumulator plants: a review of the ecology and physiology of a biological resource for phytoremediation of metal-polluted soils, in: Phytoremediation of Contaminated Soil and Water, CRC Press, Boca Raton, FL, 2000, pp. 85-107.

[20] M. Zhao, et al., Rhizosphere bacteria enhance selenium accumulation and volatilization by Indian mustard, Plant Physiol. 119 (1999) 565-573.

[21] S.G. Yang, et al., Experiment on Eisenia foetida for pre-compost of chook manure, Chin. J. Eco-agri. 15 (2007) 55-57.

[22] D. Gleba, et al., Use of plant roots for phytoremediation and molecular farming, Proc. Natl. Acad. Sci. U.S.A. 96 (1999) 5973-5977.

[23] M. Geoffrey, et al., Microorganisms in toxic metal-polluted soils, in: Soil Biology, vol. 3: Microorganisms in Soils: Roles in Genesis and Functions, Springer, Berlin, 2006, pp. 1-69.

[24] B.J.J. Lugtenberg, et al., Microbial stimulation of plant growth and protection from disease, Curr. Opin. Biotechnol. 2 (1991) 457-464.

[25] Y. Korshunova, et al., The IRT1 protein from Arabidopsis thaliana, Plant Mol. Biol. 40 (1999) 37-44.

[26] R.S. Dubery, et al., Heavy metal uptake and detoxification mechanism in plants, Int. J. Agri. Res. 1 (2006) 122-141.

[27] S. Yuebing, et al., Phytoremediation and strengthening measures for soil contaminated by heavy metals, Chin. J. Environ. Eng. 1 (2007) 23-28.

[28] Begley, et al., Mechanistic studies of a protonolytic organomercurial cleaving enzyme: bacterial organomercurial lyase, Biochemistry 25 (1986) 7192-7200.

[29] C.L. Rugh, et al., Development of transgenic yellow poplar for mercury phytoremediation, Nat. Biotechnol. 16 (1998) 925-928.

[30] A.C.P. Heaton, et al., Phytoremediation of mercury- and methylmercurypolluted soils using genetically engineered plants, J. Soil Contam. 7 (1998) 497-509.

[31] R. Bizili, et al., Phytodetoxification of hazardous organomercurials by genetically engineered plants, Nat. Biotechnol. 18 (2000) 213-217.

[32] O.N. Ruiz, et al., Phytoremediation of organomercurial compounds via chloroplast genetic engineering, Plant Physiol. 132 (2003) 1344-1352.

[33] S. Lee, et al., Overexpression of Arabidopsis phytochelatin synthase paradoxically leads to hypersensitivity to cadmium stress, Plant Physiol. 131 (2003) 656-663.

[34] S. Hwang, et al., Overexpression of ATP sulfurylase in Indian mustard leads to increased selenate uptake, reduction, and tolerance, Plant Physiol. 119 (1999) 123-132.
[35] A.L Wangeline, et al., Overexpression of ATP sulfurylase in Indian mustard: effects on tolerance and accumulation of twelve metals, J. Environ. Qual. 33 (2004) 54-60.

[36] S. Misra, et al., Heavy metal tolerant transgenic Brassica napus L. and Nicotiana tabacum L. plants, Theor. Appl. Genet. 78 (1989) 161-168.

[37] A. Pan, et al., Alpha-domain of human metallothionein I-A can bind to metals in transgenic tobacco plants, Mol. Gen. Genet. 242 (1994) 666-674.

[38] V. Huysen, et al., Overexpression of cystathionine-gamma-synthase enhances selenium volatilization in Brasica juncea, Planta 218 (2003) 71-78.

[39] M. Noji, et al., Cysteine synthase overexpression in tobacco confers tolerance to sulfur-containing environmental pollutants, Plant Physiol. 126 (2001) 973-980.

[40] Y.L. Zhu, et al., Overexpression of gluthatione synthetase in Indian mustard enhances cadmium accumulation and tolerance, Plant Physiol. 119 (1999) 73-79.

[41] Y.L. Zhu, et al., Cadmium tolerance and accumulation in Indian mustard is enhanced by overexpressing $\gamma$-glutamilcysteine synthetase, Plant Physiol. 121 (1999) 1169-1177.

[42] Arisi, et al., Responses to cadmium in leaves of transformed poplars overexpressing $\gamma$-glutamylcysteine synthatase, Physiol. Plant 109 (2000) 143149.

[43] T. Arazi, et al., A tobacco plasma membrane calmodulin-binding transporter confers $\mathrm{Ni}^{2+}$ tolerance and $\mathrm{Pb}^{2+}$ hypersensitivity in transgenic plants, Plant $\mathrm{J}$. 20 (1999) 171-182.

[44] M. Pilon, et al., Enhanced selenium tolerance and accumulation in transgenic Arabidopsis expressing a mouse selenocysteine lyase, Plant Physiol. 131 (2003) 1250-1257.

[45] C. Gisbert, et al., A plant genetically modified that accumulates $\mathrm{Pb}$ is especially promising for phytoremediation, Biochem. Biophys. Res. Commun. 303 (2003) $440-445$.

[46] W.Y. Shi, H.B. Shao, H. Li, M.A. Shao, S. Du, Co-remediation of the lead-polluted garden soil by exogenous natural zeolite and humic acids, J. Hazard. Mater. 167 (2009) 136-140.

[47] P.G. Yang, R.Z. Mao, H.B. Shao, Y.F. Gao, An investigation on the distribution of eight hazardous heavy metals in the suburban farmland of China, J. Hazard. Mater. 167 (2009) 1246-1251.

[48] H. Li, W.Y.Shi, H.B. Shao, M.A. Shao, The remediation of the lead-polluted garden soil by natural zeolite, J. Hazard. Mater. 169 (2009) 1106-1111.

[49] H.B. Shao, L.Y. Chu, Z.H. Lu, C.M. Kang, Primary antioxidant free radical scavenging and redox signaling pathways in higher plant cells, Int. J. Biol. Sci. 4 (2008) 8-14.

[50] H.B. Shao, L.Y. Chu, C.A. Jaleel, P. Manivannan, R. Panneerselvam, M.A. Shao Understanding water deficit stress-induced changes in the basic metabolism of higher plants-biotechnologically and sustainably improving agriculture and the eco-environment in arid regions of the globe, Crit. Rev. Biotech. 29 (2009) 131-151.

[51] X.L. Wang, X.Q. Ma, Advance in the research of phytoremediation in heavy metal contaminated soils, Subtrop. Agri. Res. 4 (2008) 44-49. 